\title{
Chronic Administration of Bovine Milk-Derived $\alpha$-Lactalbumin Improves Glucose Tolerance via Enhancement of Adiponectin in Goto-Kakizaki Rats with Type 2 Diabetes
}

\author{
Makoto Yamaguchi* and Shoko Takai \\ Food Science Research Labs, R\&D Div., Meiji Co., Ltd.; 540 Naruda, Odawara, Kanagawa 250-0862, Japan.
}

Received September 30, 2013; accepted December 16, 2013

Inflammation has been implicated in development of the insulin resistance that leads to elevated blood sugar levels associated with type 2 diabetes. It is reported that salsalate, a common non-steroidal antiinflammatory drug, has been shown to decrease blood glucose concentration in some clinical study. Recently, we found that bovine milk-derived $\alpha$-lactalbumin had anti-inflammatory activity caused by inhibiting cyclooxygenase-2. In this study, we investigated the effects of chronic administration of $\alpha$-lactalbumin on glucose tolerance in Goto-Kakizaki (GK) rats, a model of type 2 diabetes. After 10 weeks administration of the $\alpha$-lactalbumin $(300 \mathrm{mg} / \mathrm{kg}$, twice a day), oral glucose tolerance tests revealed significant decrements of blood glucose levels after glucose loading. However, significant differences of insulin levels were not observed among three GK rats groups after glucose loading. $\alpha$-Lactalbumin treatment enhanced high molecular weight form of adiponectin and suppressed prostaglandin E2 levels in plasma. These results suggest that $\alpha$-lactalbumin effectively decreased blood glucose levels after glucose loading in GK rat, and the decrements may be due to enhancement of adiponectin.

Key words $\alpha$-lactalbumin; diabetes; adiponectin; cyclooxygenase; prostaglandin

Type 2 diabetes mellitus (formerly noninsulin-dependent diabetes mellitus (NIDDM)) is a metabolic disorder that is characterized by high blood glucose in the context of insulin resistance and relative insulin deficiency. A number of lifestyle factors are known to be important to the development of type 2 diabetes, including: obesity (defined by a body mass index of greater than thirty), lack of physical activity, poor diet, stress, and urbanization. ${ }^{1)}$ Dietary factors also influence the risk of developing type 2 diabetes. Consumption of sugarsweetened drinks in excess is associated with an increased risk. $^{2)}$

Components of the immune system are altered in obesity and type 2 diabetes, with the most apparent changes occurring in adipose tissue, the liver, pancreatic islets, the vasculature and circulating leukocytes. ${ }^{3)}$ These immunological changes include altered levels of specific cytokines and chemokines, changes in the number and activation state of various leukocyte populations and increased apoptosis and tissue fibrosis. Together, these changes suggest that inflammation participates in the pathogenesis of type 2 diabetes.

Salsalate, a nonacetylated prodrug of salicylate, has shown potential for decreasing blood glucose concentrations by decreasing inflammation in several clinical studies. ${ }^{4)}$ These results support this notion and have opened the door for immunomodulatory strategies for the treatment of type 2 diabetes that simultaneously lower blood glucose levels and potentially reduce the severity and prevalence of the associated complications of this disease. However, the use of non-steroidal antiinflammatory drugs (NSAIDs) for the purpose of prevention of diabetes is not recommended, because the prolonged use of NSAIDs is associated with unacceptable side effects. ${ }^{5}$

On the other hands, two to three servings of low-fat milk (or other low-fat dairy food such as cheese and yogurt) each day are recommended by the American Diabetes Association

The authors declare no conflict of interest.
(ADA): "Including sources of dairy products in your diet is an easy way to get calcium and high-quality protein." Milk products have the ability to enhance the insulin response when supplied in a mixed meal.

Milk is one of the oldest functional foods available to mammals. From birth, mammals rely on mother's milk for nutrition and immune protection. Scientists are beginning to develop an understanding of the various components of milk, including whey, and how they may impact health and disease. $\alpha$-Lactalbumin, $\beta$-lactoglobulin, and lactoferrin, which are major protein components of bovine milk whey, have been known to have many pharmacological functions such as an antioxidant, ${ }^{6)}$ antihypertensive, ${ }^{7)}$ hypolipidemic, ${ }^{8)}$ antiviral, ${ }^{9)}$ antibacterial, ${ }^{10)}$ chelating, ${ }^{11)}$ hypoallergenic ${ }^{12)}$ and antitumor agent. ${ }^{13)}$ We have confirmed that lactoferrin protects tumor necrosis factor- $\alpha$ (TNF- $\alpha$ ) production caused by sensitization of hepatic monocytes (kupffer cells) by lipopolisaccaride in C57BL/6J mice. ${ }^{14)}$ We also reported that oral administered bovine milk-derived $\alpha$-lactalbumin $(300 \mathrm{mg} / \mathrm{kg}) \mathrm{had}$ anti-inflammatory ${ }^{15)}$ and anti-nociceptive activity by inhibiting cyclooxygenase-2 $(\mathrm{COX}-2)^{16)}$ in rat.

The aim of the present study was to assess two points: 1) Can dietary treatment with $\alpha$-lactalbumin improve impaired glucose tolerance? 2) If so, what is the mechanism of action of $\alpha$-lactalbumin against impaired glucose tolerance? In this study, we used Goto-Kakizaki (GK) rats, a model of type 2 diabetes without obesity.

\section{MATERIALS AND METHODS}

Reagents $\alpha$-Lactalbumin (BioPURE Alphalactalbumin ${ }^{\mathrm{TM}}$ ) was obtained from Davisco Foods International Inc. (Eden Prairie, MN, U.S.A.). Salsalate was purchased from Tokyo Chemical Industries, Co., Ltd. (Tokyo, Japan). Glucose was purchased from Wako Pure Chemical Industries, Ltd. (Osaka, Japan). Sample solutions were prepared by dissolving drugs in 
distilled water. In the normal and control rats, distilled water was administered instead of sample solution.

Animal Experiments Male Wistar and GK rats at 4 weeks of age were obtained from SLC Japan, Inc. (Shizuoka, Japan) and housed in plastic cages for 1 week prior to commencement of experiments under controlled temperature (22士 $\left.1{ }^{\circ} \mathrm{C}\right)$, humidity $(55 \pm 15 \%)$, and 12 -h light/dark cycle, and the light period was 7:00 to 19:00. All animal experiments were performed in accordance with "The guidelines of the Meiji Co., Ltd. for the care and use of laboratory animals."

GK rats were divided into three groups $(n=6)$ : 1) vehicle (Control) group; 2) $\alpha$-lactalbumin (300 mg/kg)-treated group; 3) salsalate $(25 \mathrm{mg} / \mathrm{kg})$-treated group. A test sample or vehicle (distilled water) was given orally in a volume of $10 \mathrm{~mL} / \mathrm{kg}$ through a stainless tube attached to a $2.5 \mathrm{~mL}$ sylinge twice a day for 10 weeks. Wistar rats (normal group) were given distilled water orally in a volume of $10 \mathrm{~mL} / \mathrm{kg}$ twice a day for 10 weeks. After 10 weeks chronic administration of sample, $10 \%$ glucose solution $(1 \mathrm{~g} / \mathrm{kg}$ body weight) was orally administered in the fasting state. Blood samples were obtained from tail vein at $0,15,30,60$ and $120 \mathrm{~min}$ after glucose administration. The blood glucose concentration was measured with BREEZE 2 (Bayer Yakuhin, Ltd., Osaka, Japan). Plasma was collected and frozen at $-80^{\circ} \mathrm{C}$ for determination of insulin levels. After $2 \mathrm{~d}$ of glucose tolerance test, rats were killed by exsanguinations from the descending vena cava. Blood was centrifuged for $10 \mathrm{~min}$ at $12000 \times \mathbf{g}$. Plasma was then collected and frozen at $-80^{\circ} \mathrm{C}$.

Enzyme Immunoassay Levels of insulin and high molecular weight form of adiponectin were determined in the plasma using an enzyme-linked immunosorbent assay (ELISA) kits from SHIBAYAGI Co., Ltd. (Gunma, Japan), following the manufacturer's protocol. For the determination of prostaglandin E2 (PGE2) levels, the plasma samples were mixed with ethyl acetate, vortexed for $3 \mathrm{~min}$, and then centrifuged at $12000 \times \boldsymbol{g}$ for $10 \mathrm{~min}$. The organic layer was collected and dried using nitrogen gas. The dried samples were then reconstituted in deionized water, and levels of PGE2 were determined using EIA kits from R\&D systems Inc.

Statistical Analyses All data are expressed as means S.E.M.. Fisher's Protected Least Significant Difference test (parametric test) or Mann-Whitney's $U$-test (non-parametric test) were performed. Difference with $p<0.05(*)$ and $p<0.01(* *)$ were considered as statistically significant.

\section{RESULTS}

Effect of $\boldsymbol{\alpha}$-Lactalbumin on General Health The body weights of $\alpha$-lactalbumin supplemented groups were similar to those of the control (vehicle) supplemented group throughout the experiment (Fig. 1). The $\alpha$-lactalbumin treated rats looked healthy throughout the experiment and no signs of toxicity were observed.

Effect of $\alpha$-Lactalbumin on Blood Glucose Levels in $(1 \mathrm{~g} /$ kg) Oral Glucose Tolerance Test in Rats The results of the oral glucose tolerance test are shown in Fig. 2. After 10 weeks administration of $\alpha$-lactalbumin, glucose intolerance was observed in GK rat groups as compared with the age-matched normal Wistar rat group. In GK rats groups, blood glucose levels of $\alpha$-lactalbumin supplemented group were significantly lowers those of control group at 30 and $60 \mathrm{~min}$ after oral glu-

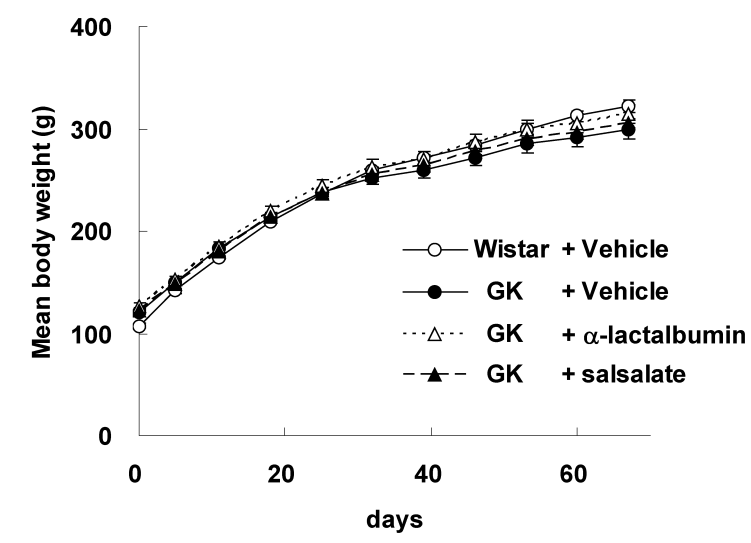

Fig. 1. Mean Body Weights of Male Rats during the Study

For 10 weeks, a test sample or vehicle (distilled water) were given orally twice a day in GK and Wistar rats. Data are presented as means of 6 rats; bars, \pm S.E.M.

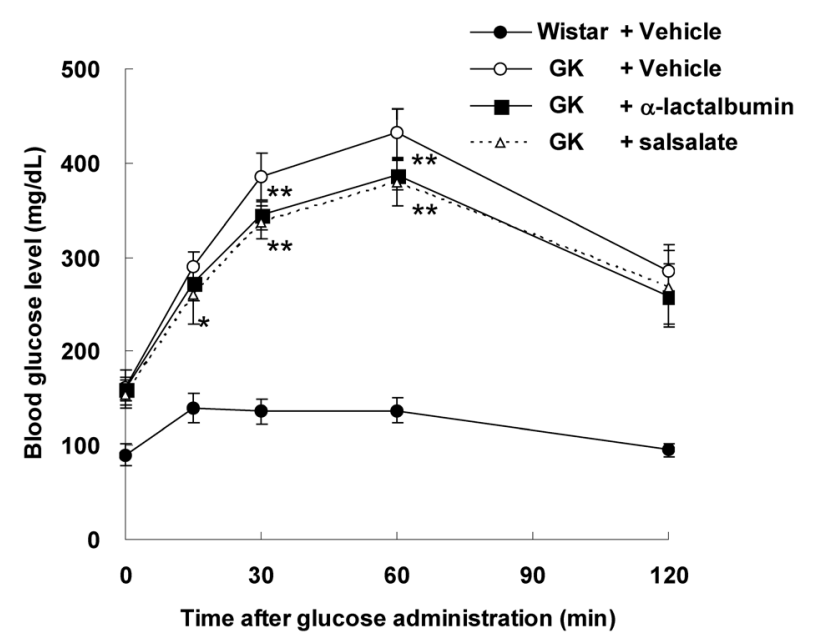

Fig. 2. Effect of $\alpha$-Lactalbumin on Blood Glucose Levels after Oral Glucose Administration in Rats

Oral glucose tolerance was determined in GK and Wistar rats after 10 weeks administration of $\alpha$-lactalbumin or salsalate. Values represent the mean \pm S.E.M. of 6 rats. $* * *$ Significant difference from the GK control group $(p<0.05,0.01)$.

cose administration. Blood glucose levels of salsalate supplemented group were significantly lower those of control group at 15,30 and $60 \mathrm{~min}$ after oral glucose administration.

Effect of $\alpha$-Lactalbumin on Blood Insulin Levels in $(1 \mathrm{~g} /$ kg) Oral Glucose Tolerance Test in Rats The results of the blood insulin levels are shown in Fig. 3. Impaired insulin secretion was observed in GK rat groups as compared with the age-matched normal Wistar rat group. However, no significant differences were observed among the three GK rat groups.

Changes in High Molecular Weight Form of Adiponectin Levels in Plasma of $\alpha$-Lactalbumin Supplemented Rats The results of the blood high molecular weight form of adiponectin levels are shown in Fig. 4. The plasma levels of high molecular weight form of adiponectin were similar in both control GK rats $(1.02 \pm 0.20 \mu \mathrm{g} / \mathrm{mL})$ and normal Wistar rats $(0.88 \pm 0.15 \mu \mathrm{g} / \mathrm{mL})$. In GK rats group, treatment with $\alpha$-lactalbumin caused a significant increased high molecular weight form of adiponectin $(1.86 \pm 0.70 \mu \mathrm{g} / \mathrm{mL} ; p<0.01 v s$. vehicle). Salsalate treatment significantly increased high molecular weight form of adiponectin $(2.10 \pm 0.18 \mathrm{pg} / \mathrm{mL} ; p<0.01$ vs. vehicle).

Changes in PGE2 Levels in Plasma of $\alpha$-Lactalbumin 


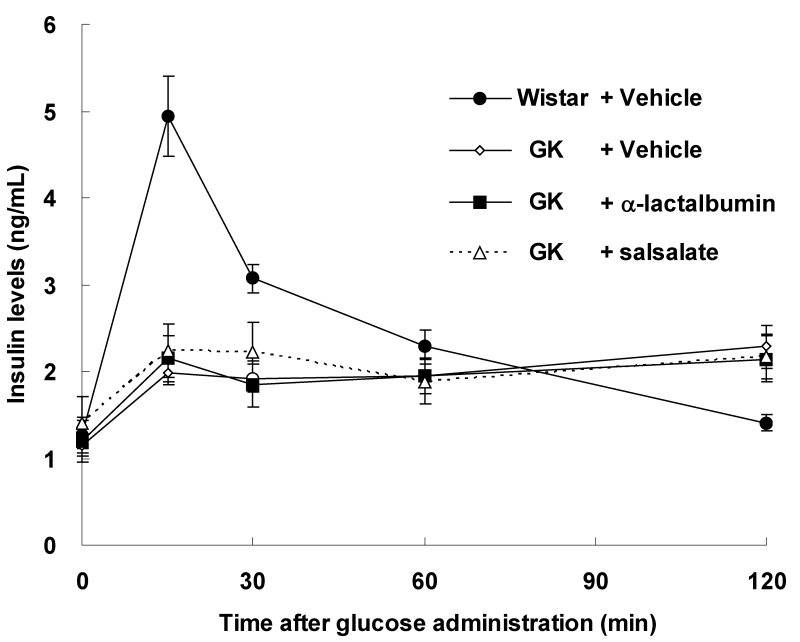

Fig. 3. Effect of $\alpha$-Lactalbumin on Blood Insulin Levels after Oral Glucose Administration in Rats

Insulin secretion was determined in GK and Wistar rats after 10 weeks administration of $\alpha$-lactalbumin or salsalate. Values represent the mean \pm S.E.M. of 6 rats.

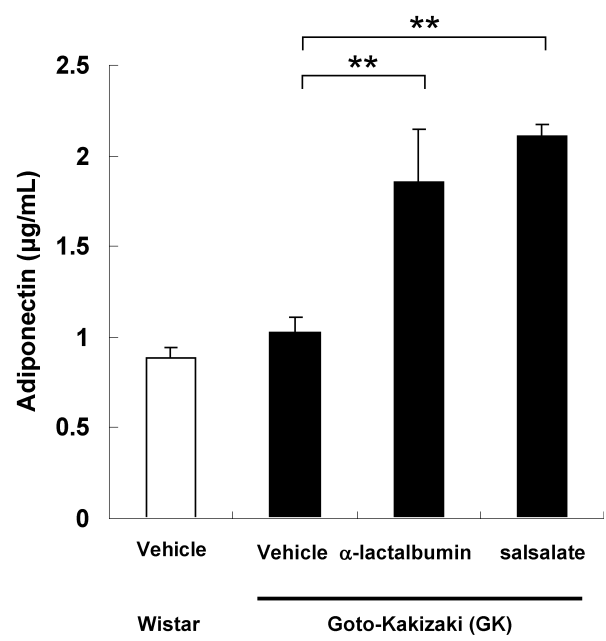

Fig. 4. Changes in High Molecular Weight Form of Adiponectin Levels in Plasma of $\alpha$-Lactalbumin Supplemented Rats

High molecular weight form of adiponectin levels in plasma were determined in GK and Wistar rats after 10 weeks administration of $\alpha$-lactalbumin or salsalate. Values represent the mean \pm S.E.M. of 6 rats. ${ }^{* *}$ Significant difference from the GK control group $(p<0.01)$

Supplemented Rats The results of the blood PGE2 levels are shown in Fig. 5. The plasma levels of PGE2 in control GK rats $(908.1 \pm 382.1 \mathrm{pg} / \mathrm{mL})$ were higher than in normal Wistar rats $(146.9 \pm 81.9 \mathrm{pg} / \mathrm{mL})$. In GK rats group, treatment with

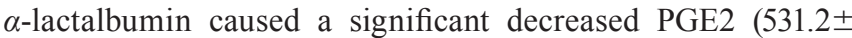
$172.0 \mathrm{pg} / \mathrm{mL} ; p<0.05$ vs. vehicle). Salsalate treatment significantly decreased PGE2 $(259.6 \pm 212.2 \mathrm{pg} / \mathrm{mL} ; p<0.01$ vs. vehicle).

\section{DISCUSSION}

Milk is an opaque white liquid produced by the mammary glands of female mammals. Cow milk contains, on average, $3.4 \%$ protein, $3.6 \%$ fat, and $4.6 \%$ lactose, $0.7 \%$ minerals and supplies $66 \mathrm{kcal}$ of energy per $100 \mathrm{~mL}$. $\alpha$-Lactalbumin exist approximately $120 \mathrm{mg}$ in cow's milk $(100 \mathrm{~mL})$, and is also present in the milk of many other mammalian species.

In our previous study, the glucose intolerance ameliorating

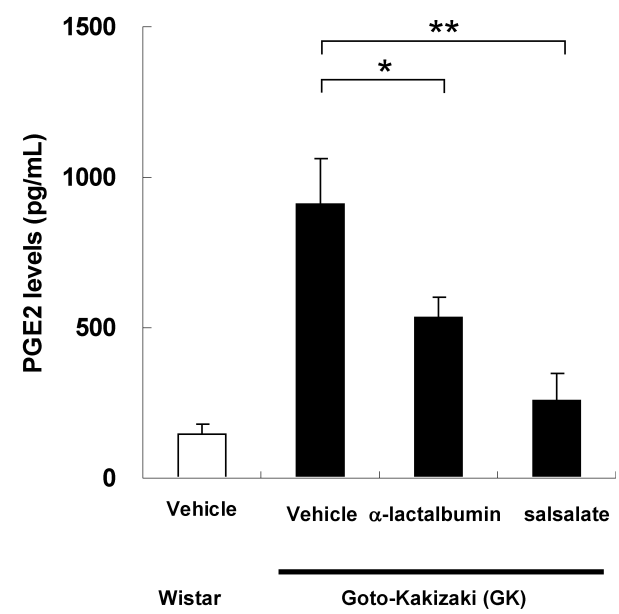

Fig. 5. Changes in PGE2 Levels in Plasma of $\alpha$-Lactalbumin Supplemented Rats

PGE2 levels in plasma were determined in GK and Wistar rats after 10 weeks administration of $\alpha$-lactalbumin or salsalate. Values represent the mean \pm S.E.M. of 6 rats. $* * *$ Significant difference from the GK control group $(p<0.05,0.01)$

effect of $\alpha$-lactalbumin was not observed at 5 weeks of administration (unpublished data). In contrast, the results of this study indicated that 10 weeks administration of $\alpha$-lactalbumin has a marked ameliorating effect against glucose intolerance in GK rats (Fig. 2). This datum may support the report that milk proteins, in particular the whey fraction, have the ability to enhance the insulin response. ${ }^{17)}$ It has been reported that glucose concentrations in GK rats showed a biphasic increase. ${ }^{18)}$ The first phase started at approximately 5 weeks, resulting in a rise of glucose by 15 to $25 \mathrm{mg} / \mathrm{dL}$, and the second phase appeared at 14 to 15 weeks with a glucose upsurge of more than $100 \mathrm{mg} / \mathrm{dL}$. These results indicate that mild-tomoderate diabetes was sustained in GK rats during our experimental period.

In this study, we measured the insulin level of each rat. Although plasma insulin levels in the groups of $\alpha$-lactalbumin supplemented- or salsalate suppulemented-rats were slightly higher than those of the control group at $15 \mathrm{~min}$ after glucose injection, significantly differences were not observed among the three GK rats groups (Fig. 3). These results suggested that insulin might not be involved in the ameliorating effects of $\alpha$-lactalbumin on glucose intolerance in GK rats.

In this study, the fasting blood glucose levels, which were the markers related to diabetes, were determined every week, but $\alpha$-lactalbumin and salsalate had no effects on fasting blood levels of glucose (data not shown). In the first place, the GK rat has developed on the basis of selective breeding of animals with a positive oral glucose tolerance test (OGTT). Besides, mild-to-moderate diabetes was sustained in GK rats during our experimental period as described above. These results indicate that enhancement of adiponectin by $\alpha$-lactalbumin might contributes to improvement of transient hyperglycemia.

The present study showed that the plasma adiponectin levels were significantly higher in $\alpha$-lactalbumin or salsalate administered rat than GK control rat (Fig. 4). Adiponectin is a hormone secreted by adipocytes and acts as adipokine in muscle and liver which increases fatty acid oxidation and glucose uptake. Although the mechanism of regulation of plasma adiponectin is unknown, it has been shown that circulating adiponectin concentrations are (i) not affected by fasting or leptin 
administration $^{19)}$; (ii) correlated with insulin sensitivity ${ }^{20)}$; (iii) increased with the administration of peroxisome proliferatoractivated receptor $\gamma(\operatorname{PPAR} \gamma)$ ligand, thiazolidinedione ${ }^{21)}$; and (iv) increased when weight is lost. ${ }^{22}$ Adipose tissue inflammation induces systemic insulin resistance in persons with obesity and heart failure, and has a crucial role in the progression of these diseases. ${ }^{23)}$ Chronic inflammatory processes share a common mechanism in which increased production of reactive oxygen species activates p53 and nuclear factor-kappa B (NF$\kappa \mathrm{B})$ signaling, leading to up-regulation of pro-inflammatory cytokine expression and impairment of glucose metabolism. We already reported that oral administered bovine milk-derived $\alpha$-lactalbumin had anti-inflammatory activity inhibiting by COX-2. ${ }^{16)}$ Recently, it has been reported that dairy protein affect expression of PPAR $\gamma$ mRNA $^{24,25)}$ From these results, $\alpha$-lactalbumin suppressed adipose tissue inflammation and the ameliorating effects of $\alpha$-lactalbumin on glucose intolerance in GK rats might be mostly caused by enhancement of adiponectin. In addition, $\alpha$-lactalbumin might improve insulin sensitivity via enhancement of adiponection in peripheral tissues, but we have no data about glucose uptake in peripheral tissued or hepatic glycogenesis induced by insulin. On this points, further study was needed.

In an animal model of type 2 diabetes, chronic inflammation plays an etiological role. Chronic activation of the immune system causes a decrease in insulin sensitivity, which may contribute to the pathogenesis of type 2 diabetes. The present results suggest that chronic inflammatory responses are involved in the decrease of pancreatic $\beta$-cells via the induction of COX-2 and PGE2, which leads to an increased susceptibility to type 2 diabetes. ${ }^{26)}$ Although COX-2 is an inducible enzyme in most tissues, it has been shown to be expressed constitutively in pancreatic islets. Therefore, it is probable that an increase in the basal level of COX-2 expression, caused by a promoter polymorphism, enhances the susceptibility to type 2 diabetes via the suppression of the renewing capabilities of islet $\beta$-cells. However, it is reported that the pathophysiologic changes of pancreas occur from the late fetal period in GK rat. As compared to Wistar controls, GK fetuses exhibited higher plasma glucose level and lower plasma insulin level. ${ }^{27)}$ From these results, the ameliorating effects of $\alpha$-lactalbumin on glucose intolerance in adult GK rats might be slightly caused by suppression of PGE2. Inhibiting the production of prostaglandins maybe contribute to the prevention of diabetes complications, such as peripheral neuropathy, cardiovascular disorders and renal failure.

Non-steroidal anti-inflammatory drugs (NSAIDs) bring symptomatic relief many patients with arthritic, inflammatory or other conditions; however, the benefits of these drugs must be balanced against the upper gastrointestinal (GI) side-effects associated with their use. ${ }^{28}$ By inhibiting COX-1, NSAIDs increase the risk of a peptic ulcer developing and bleeding. On the other hand, we reported that $\alpha$-lactalbumin showed selectivity on COX-2 as compared with COX-1. ${ }^{15}$ Additionally, it has been reported that $\alpha$-lactalbumin fortifies the mucus gel layer by stimulating mucin production and secretion in gastric mucus-producing cells, and that this enhancing effect is independent of endogenous PGE2. ${ }^{29)}$ These results indicated that $\alpha$-lactalbumin reduces the gastrointestinal side-effects.

Interleukin-1 $\beta$ (IL-1 $\beta$ ) is a regulator of the body's inflammatory response and is produced after infection, injury, and antigenic challenge. It plays a role in various diseases, including autoimmune diseases such as rheumatoid arthritis, inflammatory bowel diseases and type 1 diabetes, as well as in diseases associated with metabolic syndrome such as atherosclerosis, chronic heart failure and type 2 diabetes. ${ }^{30}$ Macrophages are the primary source of IL-1, but epidermal, epithelial, lymphoid and vascular tissues also synthesize IL-1. In general, IL- $1 \beta$ production and secretion have also been reported from pancreatic islets. Insulin-producing beta-cells within pancreatic islets are specifically prone to IL- $1 \beta$-induced destruction and loss of function. In this study, the plasma the levels of IL- $1 \beta$ were similar among the three GK rat groups (data not shown), because pathophysiologic changes of pancreas occur from the late fetal period in GK rat. These results suggested that IL-1 $\beta$ might not be involved in the ameliorating effects of $\alpha$-lactalbumin on glucose intolerance in GK rats. On this point, further experiment would be needed.

In conclusion, we have reported ameliorating effect of $\alpha$-lactalbumin against glucose intolerance: the amelioration may be due to enhancement of adiponectin. These results suggest that long-term consumption of $\alpha$-lactalbumin is a safe and useful natural drug to reduce the risk of type 2 diabetes.

Acknowledgments The authors thank Mr. Takeshi Mori for constant advice and valuable discussion. We have been stimulated by the knowledge of the general experimental results and ideas of Dr. Masayuki Uchida. We also thank Dr. Taketo Yamaji and Dr. Hiroyuki Itoh for providing encouragement throughout the study.

\section{REFERENCES}

1) Williams Textbook of Endocrinology, 12th ed., Elsevier/Saunders, Philadelphia, pp. 1371-1435 (2011).

2) Malik VS, Schulze MB, Hu FB. Intake of sugar-sweetened beverages and weight gain: a systematic review. Am. J. Clin. Nutr., 84, 274-288 (2006).

3) Donath MY, Shoelson SE. Type 2 diabetes as an inflammatory disease. Nat. Rev. Immunol., 11, 98-107 (2011)

4) Goldfine AB, Silver R, Aldhahi W, Cai D, Tatro E, Lee J, Shoelson SE. Use of salsalate to target inflammation in the treatment of insulin resistance and type 2 diabetes. Clin. Transl. Sci., 1, 36-43 (2008).

5) Bjarnason I, Hayllar J, MacPherson AJ, Russell AS. Side effects of nonsteroidal anti-inflammatory drugs on the small and large intestine in humans. Gastroenterology, 104, 1832-1847 (1993).

6) Brown EC, DiSilvestro RA, Babaknia A, Devor ST. Soy versus whey protein bars: effects on exercise training impact on lean body mass and antioxidant status. Nutr. J., 3, 22 (2004).

7) Saito T. Antihypertensive peptides derived from bovine casein and whey proteins. Adv. Exp. Med. Biol., 606, 295-317 (2008).

8) Marshall K. Therapeutic applications of whey protein. Altern. Med. Rev., 9, 136-156 (2004).

9) Neurath AR, Li YY, Strick N, Jiang S. A herpesvirus inhibitor from bovine whey. Lancet, 347, 1703-1704 (1996).

10) Shah NP. Effects of milk-derived bioactives: an overview. Br. J. Nutr., 84 (Suppl. 1), S3-S10 (2000).

11) Hurrell RF, Lynch SR, Trinidad TP, Dassenko SA, Cook JD. Iron absorption in humans as influenced by bovine milk proteins. Am. J. Clin. Nutr., 49, 546-552 (1989).

12) Shimizu N, Dairiki K, Ogawa S, Kaneko T. Dietary whey protein hydrolysate suppresses development of atopic dermatitis-like skin lesions induced by mite antigen in $\mathrm{NC} / \mathrm{Nga}$ mice. Allergol. Int., 55, 
185-189 (2006).

13) Bounous $G$, Batist $G$, Gold P. Whey proteins in cancer prevention. Cancer Lett., 57, 91-94 (1991).

14) Yamaguchi M, Matsuura M, Kobayashi K, Sasaki H, Yajima T, Kuwata T. Lactoferrin protects against development of hepatitis caused by sensitization of Kupffer cells by lipopolysaccharide. Clin. Diagn. Lab. Immunol., 8, 1234-1239 (2001).

15) Yamaguchi M, Uchida M. Alpha-lactalbumin suppresses interleukin-6 release after intestinal ischemia/reperfusion via nitric oxide in rats. Inflammopharmacology, 15, 43-47 (2007).

16) Yamaguchi M, Yoshida K, Uchida M. Novel functions of bovine milk-derived alpha-lactalbumin: anti-nociceptive and anti-inflammatory activity caused by inhibiting cyclooxygenase- 2 and phospholipase A2. Biol. Pharm. Bull., 32, 366-371 (2009).

17) Frid AH, Nilsson M, Holst JJ, Björck IM. Effect of whey on blood glucose and insulin responses to composite breakfast and lunch meals in type 2 diabetic subjects. Am. J. Clin. Nutr., 82, 69-75 (2005).

18) Cao Y, Dubois DC, Sun H, Almon RR, Jusko WJ. Modeling diabetes disease progression and salsalate intervention in Goto-Kakizaki rats. J. Pharmacol. Exp. Ther., 339, 896-904 (2011).

19) Gavrila A, Chan JL, Yiannakouris N, Kontogianni M, Miller LC, Orlova C, Mantzoros CS. Serum adiponectin levels are inversely associated with overall and central fat distribution but are not directly regulated by acute fasting or leptin administration in humans: cross-sectional and interventional studies. J. Clin. Endocrinol. Metab., 88, 4823-4831 (2003).

20) Weyer C, Funahashi T, Tanaka S, Hotta K, Matsuzawa Y, Pratley RE, Tataranni PA. Hypoadiponectinemia in obesity and type 2 diabetes: close association with insulin resistance and hyperinsulinemia. J. Clin. Endocrinol. Metab., 86, 1930-1935 (2001).

21) Maeda N, Takahashi M, Funahashi T, Kihara S, Nishizawa $H$, Kishida K, Nagaretani H, Matsuda M, Komuro R, Ouchi N, Kuri- yama H, Hotta K, Nakamura T, Shimomura I, Matsuzawa Y. PPARgamma ligands increase expression and plasma concentrations of adiponectin, an adipose-derived protein. Diabetes, 50, 2094-2099 (2001).

22) Pešić V, Janac B, Milanovic D, Tomic M, Veskov R, Rakic L. Motor effects of amphetamine in rats pretreated with either dizocilpine or phencyclidine. Behav. Brain Res., 147, 211-215 (2003).

23) Shimizu I, Yoshida Y, Katsuno T, Minamino T. Adipose tissue inflammation in diabetes and heart failure. Microbes Infect., 15, 11-17 (2013).

24) Eller LK, Reimer RA. Dairy protein attenuates weight gain in obese rats better than whey or casein alone. Obesity (Silver Spring), 18, 704-711 (2010).

25) Rajic A, Dhulia J, Hosking CG, Autelitano DJ. A novel dairyderived isolate that inhibits adipogenesis and significantly reduces weight gain in a high fat animal model. Int. Dairy J., 20, 480-486 (2010).

26) Robertson RP. Dominance of cyclooxygenase-2 in the regulation of pancreatic islet prostaglandin synthesis. Diabetes, 47, 1379-1383 (1998).

27) Movassat J, Saulnier C, Serradas P, Portha B. Impaired development of pancreatic beta-cell mass is a primary event during the progression to diabetes in the GK rat. Diabetologia, 40, 916-925 (1997).

28) Bjarnason I, Hayllar J, MacPherson AJ, Russell AS. Side effects of nonsteroidal anti-inflammatory drugs on the small and large intestine in humans. Gastroenterology, 104, 1832-1847 (1993).

29) Ushida Y, Shimokawa Y, Toida T, Matsui H, Takase M. Bovine alpha-lactalbumin stimulates mucus metabolism in gastric mucosa. J. Dairy Sci., 90, 541-546 (2007).

30) Kolb H, Mandrup-Poulsen T. An immune origin of type 2 diabetes? Diabetologia, 48, 1038-1050 (2005). 